\title{
LA AUTONOMÍA DE APRENDIZAJE: EL PROBLEMA TERMINOLÓGICO*
}

\author{
Marta Navarro Coy**
}

Resumen: El término 'autonomía' es un concepto con el que todo el mundo está familiarizado debido a su uso en muy variados contextos. Sin embargo, cuando tratamos de aplicarlo al ámbito del aprendizaje de lenguas surgen determinados problemas principalmente por dos motivos: a) la existencia de una diversidad de conceptos utilizados en la literatura y cuyo objetivo es aparentemente la designación de un mismo fenómeno y, b) la existencia de una amplia gama de definiciones del término 'autonomía'.

En este trabajo intentaremos aclarar estas cuestiones haciendo un breve repaso de los diferentes términos utilizados en la literatura en relación con la autonomía y ofreciendo una visión homogénea y globalizadora de lo que este concepto lleva implícito.

Palabras clave: autonomía de aprendizaje, auto-instrucción, auto-dirección, aprendizaje auto-dirigido, individualización

\begin{abstract}
The term 'autonomy' has been widely used in a variety of contexts and has therefore become a familiar concept. However, when trying to apply it to the field of language learning some problems arise mainly for two reasons: a) the existence of a variety of concepts used in the literature whose main objective is apparently the designation of this same phenomenon and $b$ ) the wide range of definitions of the term autonomy that exists. This work will try to shed some light on these issues briefly going through the different terms related to autonomy that are used in the literature and offering an homogeneous and global view of what this concept implies.
\end{abstract}

Key words: learner autonomy, self-instruction, self-direction, self-directed learning, individualization.

\section{INTRODUCCIÓN}

Las últimas décadas han sido testigo de cómo la autonomía en el ámbito del aprendizaje de lenguas se ha convertido en un término 'de moda' que, como tal, ha suscitado polémica en algunos casos y creado grandes expectativas en otros.

Una de las mayores dificultades que suelen rodear al término es la de poder ofrecer una definición globalizadora del mismo que contemple todo lo que la autonomía lleva implícita ya que son muchos los aspectos que rodean al término y muchas las interpretaciones

Fecha de recepción: octubre 2004

Fecha de aceptación y versión final: febrero 2005

** Profesora de la Unidad Central de Idiomas, Universidad Católica San Antonio;

$\triangle$ mnavarro@pdi.ucam.edu 
a que se ha visto sometido. Sin embargo, podemos hacer un recorrido por los diferentes acercamientos por parte de diversos autores, de manera que podamos contrastarlos e ir entresacando todos aquellos aspectos que nos ayuden a entender de qué hablamos cuando hablamos de autonomía. No obstante, cabría señalar que desde la década de los 70, época en que este concepto empieza a adquirir gran importancia, son muy pocos los que lo han puesto en tela de juicio debido principalmente al cambio que poco a poco ha ido experimentando el papel del individuo en la sociedad y más concretamente el del aprendiz, pasando de ser considerado un sujeto pasivo a un individuo responsable, con intereses, necesidades, objetivos y estilos de aprendizaje propios y diferentes de los de cualquier otro aprendiz.

Es también importante mencionar que cuando rastreamos en la literatura (principalmente la escrita en lengua inglesa por ser la más abundante) a la búsqueda de definiciones de autonomía, el primer problema con que nos enfrentamos es el hecho de que nos encontramos con una variedad de términos cuyo objetivo es aparentemente la designación de un mismo fenómeno. Así, encontramos principalmente cuatro términos relacionados, además, por supuesto, del que nos ocupa: la autonomía. Dichos términos son: i) auto-instrucción (self-instruction); ii) auto-dirección (self-direction); iii) aprendizaje auto-dirigido (self-directed learning); y iv) individualización (individualization). En este trabajo, intentaremos reflejar el uso que se le ha dado a estos cuatro términos que acabamos de mencionar para, a continuación, dedicar otro apartado a tratar de clarificar qué es la autonomía de aprendizaje partiendo de diversas definiciones que del término se han ofrecido. Igualmente, incluiremos una breve reflexión acerca de los motivos que abogan por la adopción de un enfoque autonomizador cuando se lleva a cabo un proceso de aprendizaje.

\section{LA DIVERSIDAD TERMINOLÓGICA}

Aunque en cierta literatura, poca para ser exactos, los autores correspondientes ofrecen definiciones o explicaciones del término en concreto que utilizan, de manera muy generalizada encontramos trabajos que tratan variados aspectos concernientes al tema que nos ocupa utilizando uno u otro término sin previamente ofrecer una definición o una simple explicación del mismo de manera que se justifique su utilización frente a los demás. A continuación, realizaremos un breve recorrido por cada uno de estos cuatro términos para intentar establecer una diferenciación entre ellos que justificara su uso en determinados casos.

\subsection{Auto-instrucción}

Dickinson (1987) ofrece la siguiente definición del término auto-instrucción al que considera 'neutral': "la etiqueta 'auto-instrucción' se usa para hacer referencia a situaciones en las que un aprendiz, con otros, o solo, trabaja sin el control directo de un profesor" (Dickinson 1987: 5). Esta definición es matizada más adelante en el mismo trabajo: “[...]

\footnotetext{
1 Toda cita que aparece en español y cuya referencia es de una obra en inglés ha sido traducida por la autora.
}

Odisea, $n^{\circ}$ 6, ISSN 1578-3820, 2005, 145-160 
hay una gama de posibilidades implícita en éste término, pero la noción de responsabilidad del aprendiz es común a toda la gama" (Dickinson 1987: 8).

En esta definición encontramos dos aspectos a destacar a la hora de establecer comparaciones entre éste y los demás términos: en primer lugar, la referencia al trabajo sin el control del profesor y, en segundo, la referencia a la responsabilidad del aprendiz, referencia que va a ser común en prácticamente la totalidad de definiciones y/o alusiones a cualquiera de los términos dentro del ámbito de la autonomía. El primero de los aspectos, es decir, el que hace referencia al trabajo sin el control de un profesor, es compartido por otros autores. Así, Little (1990), en su búsqueda de una definición del término autonomía, ofrece en primer lugar cinco negaciones, es decir, lo que la autonomía no es, siendo la primera de ellas que "autonomía no es sinónimo de auto-instrucción" (self-instruction); en otras palabras, "la autonomía no se limita al aprendizaje sin un profesor" (Little 1990: 7). También para Bosch (1996) el término auto-instrucción implica la ausencia de la figura del profesor ya que, según sus propias palabras (Bosch 1996: 18-19):

Pensar que la autonomía del aprendiz no se puede encontrar en el contexto de la enseñanza presencial es caer en el error de identificar autonomía con auto-instrucción, ya que se la relaciona exclusivamente con cuestiones de libertad de organización y se olvida que la autonomía es una cuestión de la relación psicológica entre el aprendiz y el contenido del proceso de aprendizaje.

\subsection{Auto-dirección}

En lo que respecta a la auto-dirección (self-direction), hallamos principalmente dos opiniones encontradas. Para Dickinson (1987: 16) “el término 'auto-dirección' describe una actitud en particular hacia la tarea del aprendizaje", en donde el aprendiz acepta la responsabilidad de todas las decisiones concernientes a su aprendizaje pero sin llevar a cabo necesariamente la implementación de dichas decisiones, de donde se desprende que el aprendiz auto-dirigido (self-directed learner) es aquél que "retiene la responsabilidad de su propio aprendizaje" (Carver y Dickinson 1982:15). Sin embargo, lo que Dickinson considera una actitud, para Holec es "una destreza, una capacidad, un saber-cómo" (Holec 1985: 188), existiendo por tanto un contraste entre ambas opiniones que se puede percibir con más claridad en las siguientes palabras de Dickinson con respecto al aprendiz autodirigido: "ser responsable de tu propio aprendizaje es más una actitud mental que comportarse de una forma en particular o poseer unas destrezas concretas" (Dickinson 1982: 15).

\subsection{Aprendizaje auto-dirigido}

Knowles (1975), uno de los impulsores de la autonomía, utiliza el término aprendizaje auto-dirigido (self-directed learning) para poner de manifiesto el control que el aprendiz tiene sobre la planificación del aprendizaje, un control total ya que para él, el término lleva implícito tanto la toma de decisiones, como la implementación de las mismas por parte del aprendiz. También Blue (1981), al hablar de aprendizaje auto-dirigido, hace referencia al control que el aprendiz tiene sobre su propio proceso de aprendizaje y aún 
especifica más al indicar que se trata de un proceso en el cual el aprendiz "asume la responsabilidad de todas las decisiones que conciernen a los diferentes aspectos del proceso de aprendizaje y que incluyen fijar los objetivos, determinar el contenido y los métodos de aprendizaje e incluso evaluar dicho aprendizaje, así como la organización práctica, decisión del ritmo, tiempo y lugar de aprendizaje" (p. 61). Por su parte Holec (1985) afirma que el término 'aprendizaje auto-dirigido' se refiere a "la forma en que se determinan las distintas modalidades en un programa de aprendizaje" (Holec 1985: 174) pero hay, sin embargo, quien ha llegado a cuestionar la validez de éste término, como es el caso de Dickinson (1976: 15): "The use of the term self-directed learning frequently constitutes an act of faith, and is rarely accompanied by a clear description. Where a clear description is given, however, there is frequently a marked similarity between SDL and autonomy- which would lead one to suppose that these are two names for the same phenomenon".

No obstante, esta declaración queda en cierto modo en entredicho al afirmar Dickinson en el mismo artículo que la autonomía y el aprendizaje auto-dirigido difieren en el hecho de que "la autonomía es una de las muchas posibilidades dentro del aprendizaje autodirigido. Es el límite más alto del aprendizaje auto-dirigido" (Dickinson 1976: 20). De hecho, podríamos afirmar que esta consideración de Dickinson, según la cual ambos términos designan a un mismo fenómeno, está ignorando aquello a lo que cada uno hace referencia, es decir, por un lado el hecho de que cuando hablamos de aprendizaje autodirigido estamos, sin lugar a dudas, refiriéndonos al modo en que se lleva a cabo o se organiza el aprendizaje, mientras que al hablar de autonomía, la parte implicada es únicamente el aprendiz y la actitud de éste hacia el proceso de aprendizaje.

Para definir el aprendizaje auto-dirigido Riley (1987), al igual que Blue (1981), utiliza el término "proceso" y, en una descripción paralela a la de éste, afirma que el aprendizaje auto-dirigido es "un proceso por el cual los aprendices construyen e implementan su propio programa de aprendizaje" (Riley 1987: 84).

\subsection{Individualización}

En lo que respecta al término individualización, éste se ha aplicado a tal cantidad de situaciones diversas que su utilidad ha llegado incluso a ser cuestionada por algunos autores. Dichas situaciones podrían ser agrupadas en dos. Por una parte, situaciones que son un claro reflejo de la relación profesor-alumno que durante tanto tiempo e incluso hoy en día sigue reinando en las aulas y según las cuales el aprendizaje tiene una relación de causa-efecto con la enseñanza, siendo, por tanto, el producto el único medidor de la efectividad de la metodología aplicada. Por otro lado, podríamos hablar de situaciones que guardan una estrecha relación con aspectos que se hallan implícitos en las definiciones de los demás términos a los que acabamos de hacer alusión y que, por tanto, se caracterizan por su interés en el proceso de aprendizaje y no en el producto y por su interés en la atención a la diversidad.

Los dos tipos de situaciones aludidas representan las dos interpretaciones distintas que ha sufrido el término. Los años setenta parecen haber sido testigo de cómo el término individualización se asociaba al de individualismo, caracterizándose principalmente por la elaboración, por parte del profesor, de programas que permitían al aprendiz trabajar de 
forma individual pero sin dejar a éstos la más mínima posibilidad de elección. Sin embargo, los años ochenta muestran, aunque con alguna excepción, cómo el término ha sido reinterpretado desde una nueva perspectiva: la individualización como sinónimo de personalización, la atención a la diversidad y la necesidad de que el propio aprendiz sea consciente de la existencia de dicha diversidad y la tenga en consideración para ser capaz de tomar sus propias decisiones a lo largo del proceso de aprendizaje. Dickinson (1987), a pesar de su reticencia al uso del término 'individualización', claramente pone de relieve la existencia de dicha diversidad que justifica esa personalización a la que anteriormente hacíamos referencia y que se refleja en la existencia de diversas estrategias y estilos cognitivos, en la utilización de variadas estrategias de aprendizaje, en la existencia de diferentes objetivos educativos o de variados factores que afectan a la motivación (véase Dickinson 1987: 18)

Un ejemplo que refleja la primera de las situaciones a las que anteriormente aludíamos lo encontramos en una afirmación de Logan (1973), según la cual cuando hablamos de aprendizaje individualizado hacemos referencia a la situación en la cual "el profesor proporciona materiales y actividades con las que los alumnos pueden trabajar "de forma independiente', liberando así al profesor de ocuparse de las necesidades individuales" (p.15). Resulta obvio que en tal situación la individualización no se entiende como en el segundo de los casos, ya que como señala Dickinson de forma muy significativa en referencia a la afirmación de Logan, "poco hay de nuevo en que un profesor le indique a sus alumnos que realicen los ejercicios del 15 al 25", y esto es exactamente lo que en ella se refleja. Bien es cierto que el propio Logan (1970: 17) afirmaba que "los que más se acercan a la verdadera individualización son aquellos programas que ofrecen contratos individualizados a cada estudiante (sequential learning packets) que pueden combinarse de diversas formas para diferentes alumnos."

En realidad, en lo único en que parecen diferir ambas situaciones es en el contexto en que se llevan a cabo: el aula o fuera de ella. Por lo demás, tales contratos en forma de paquetes para llevar a cabo un aprendizaje secuencial no implican en absoluto la más mínima responsabilidad por parte del aprendiz. Es cierto que en estos casos el material ha sido diseñado y escogido para atender a sus necesidades, pero en realidad no se le ha dejado al aprendiz la más mínima posibilidad de elección, todo está en los materiales que pasarían a desempeñar el papel que el profesor tiene en el aula, en otras palabras, a sustituirle. Sin duda, este tipo de situaciones presenta claramente aspectos relacionados con el paradigma conductista, ya que la instrucción sigue estando en manos ajenas al aprendiz y el énfasis sigue estando en el producto, sin que exista la más mínima preocupación por cómo tiene lugar el aprendizaje.

También Riley (1987) ofrece su versión del término individualización que está íntimamente relacionada con la que acabamos de describir. Para Riley (1987: 84) "la individualización está históricamente ligada al aprendizaje programado y basada en una rigurosa psicología del comportamiento. Poca o ninguna libertad de elección se le deja al aprendiz: es el profesor quien trata de adaptar su metodología y los materiales al aprendiz".

Como puede observarse, los aspectos que mencionábamos anteriormente, y que guardaban relación con el paradigma conductista, los trata Riley de forma explícita. Es decir, para este autor el término individualización dista mucho de ser compatible con la segunda 
de las situaciones a las que hacíamos referencia, es decir, aquélla que contempla la individualización como una consecuencia lógica de atención a la diversidad. Asimismo Chaix y O’Neil (1978, citados en Blue 1981: 61) en su definición del término reflejan el paralelismo de éste con una situación que, de nuevo, más se asemeja a la que describíamos en primer lugar, es decir, a aquélla en la que la figura autoritaria del profesor lleva las riendas de un aprendizaje en el que el propio aprendiz tiene poco o nada que decir. Siguiendo a Blue (1981: 61) nos atrevemos a indicar que la definición ofrecida por Chaix y O'Neil (1978) es la que mejor refleja este tipo de situación: "un proceso de aprendizaje que (en lo concerniente a objetivos, contenido, metodología y ritmo de aprendizaje) se adapta a un individuo en particular, teniendo en cuenta sus características individuales".

Ejemplos del segundo tipo de situación (en que se intenta desarrollar la capacidad del individuo de autodirigir el propio aprendizaje, poniendo por tanto el énfasis en el proceso, y donde el uso del término individualización lleva consigo la implicación y responsabilidad del aprendiz en su proceso de aprendizaje) pueden encontrarse en trabajos de Sturtridge (1982) y Tumposky (1982). Para Sturtridge (1982: 8-9) "la individualización implica, en primer lugar, que el profesor sea considerado como un ayudante y facilitador del aprendizaje no limitándose a enseñar un contenido que supone la enseñanza de un conocimiento estático, sino ayudando al aprendiz a aprender a aprender a la vez que aprende la lengua”. Desde este punto de vista, incluso en el aula el profesor puede abandonar su papel de poseedor del conocimiento (o 'guardián del secreto', en palabras de Illich, 1971) y fomentar la autonomía del estudiante ayudándole a adquirir destrezas que le puedan servir para enfrentarse a cualquier situación de aprendizaje fuera o incluso dentro de la propia aula. También Tumposky (1982) se refiere al profesor como un facilitador, alguien que "se ocupa de su propia sensibilidad hacia los aprendices y de sus diferencias individuales en cuanto a su estilo y ritmo de aprendizaje" (Tumposky 1982: 5). Por su parte al aprendiz "se le ha de pedir que asuma más responsabilidad en lo que respecta a su propio progreso" y asimismo "debe ser capaz de efectuar elecciones, de determinar su ritmo de aprendizaje y a menudo de auto-evaluarse; debe ser más activo en la clase, contribuyendo, sugiriendo, criticando, dando forma a la clase, ayudando a sus compañeros" (p. 5).

Una vez realizado un recorrido por la diferente terminología, podemos afirmar que, de forma general, las referencias y / o definiciones de algunos de los términos, incluido el de autonomía, pueden agruparse en dos vertientes diferentes. En primer lugar, encontraríamos una forma de hacer referencia a la modalidad del aprendizaje, es decir, a cómo éste se lleva a cabo, y en este sentido hallamos términos como auto-dirección, auto-instrucción o aprendizaje autodirigido al que, del mismo modo, podríamos denominar aprendizaje en autonomía o aprendizaje autónomo y cuyo grado de autodirección puede ser mayor o menor atendiendo a la cantidad de decisiones que el aprendiz tome por sí mismo en el proceso de aprendizaje. Por otra parte, y como veremos en el siguiente apartado, hablamos de competencia cuando hacemos referencia al término autonomía, ya que lo que este término implica es, precisamente, una competencia gradual que se manifiesta en unas destrezas o actuaciones que no son innatas y para cuya adquisición es necesario un proceso de formación en estas destrezas que integran la competencia de la autonomía. 


\section{LA AUTONOMÍA DE APRENDIZAJE: DEFINICIONES E INTERPRETACIONES}

La gran mayoría de las referencias al término autonomía que encontramos en la literatura (cf. Dickinson 1987; Holec 1981; Little 1990; Trim 1988) parecen apuntar al cambio drástico producido en el campo del aprendizaje de lenguas en las últimas décadas, un cambio que, como ya hemos señalado con anterioridad, nos muestra una visión del aprendiz completamente opuesta a la que estábamos acostumbrados. Este cambio también se aprecia con gran claridad con sólo echar un vistazo a los títulos pertenecientes a dicha literatura, donde la palabra 'aprendizaje' pasa en gran medida a sustituir a 'enseñanza', sustitución que puede traducirse en el interés que ha pasado a tenerse por el proceso de aprendizaje en detrimento del producto.

Esa dirección a la que, según indicábamos, apuntan las referencias al término autonomía se puede ver claramente reflejada en las siguientes definiciones:

The ability to take charge of one's own learning Holec (1981: 3).

Learner autonomy is esentially a matter of the learner's psychological relation to the process and content of learning. We recognise it in a wide variety of behaviours as a capacity for detachment, critical reflection, decision-making, and independent action.

Little (1990: 7)

Igualmente encontramos en la literatura muchas otras definiciones que de la misma forma ponen de manifiesto la trascendencia de aspectos como la responsabilidad, la capacidad, o la disposición del aprendiz a la hora de llevar a cabo su proceso de aprendizaje. Así, por ejemplo, para Dam (1990: 17) "la autonomía se caracteriza por la disposición a hacerse cargo del propio aprendizaje al servicio de las propias necesidades y propósitos”. En esta definición, la palabra "disposición" resulta clave en cuanto que conlleva un alto grado de implicación por parte del aprendiz. Esta disposición a hacerse cargo del propio aprendizaje, continúa Dam, "lleva consigo una capacidad y una disposición para actuar de forma independiente y en cooperación con otros como persona social responsable" (p. 17).

Como podemos observar, esta definición de Dam contempla aspectos cercanos a la definición ofrecida por Holec (1981), en la que el término "capacidad" juega un papel esencial. De igual forma, el uso de la palabra "disposición”, que comparte significado con el término "actitud”, hace que dicha definición se asemeje a aquélla que ofrecía Dickinson del término self-direction: "el término autodirección describe una actitud en particular hacia la tarea del aprendizaje en la que el aprendiz acepta la responsabilidad de todas las decisiones que tienen que ver con su aprendizaje pero no necesariamente lleva a cabo la implementación de dichas decisiones" (Dickinson 1987: 11). De nuevo, por tanto, podemos comprobar cómo en ocasiones se tiende a mezclar las diferentes vertientes a que hacíamos alusión en el punto anterior y que guardan relación bien con la modalidad del aprendizaje, o bien con la competencia del aprendiz.

Conceptos como los de capacidad o responsabilidad también están siempre presentes en los trabajos llevados a cabo en el CRAPEL (Centre de Recherche et d'Applications 
Pédagogues en Langues) de la Universidad de Nancy (Francia), donde el término autonomía es considerado una "filosofía de aprendizaje" (Stanchina 1976: 35), utilizándose para describir la forma en que allí se lleva a cabo el aprendizaje. Stanchina (1975) refleja de forma muy clara lo que para el CRAPEL lleva implícito el ejercicio de la autonomía y que se basa en el hecho de que es el aprendiz quien decide sus objetivos, su modo de aprendizaje, los materiales que utilizará y el ritmo al que los utilizará, lo cual supone un aprendizaje con un grado máximo de autodirección. Así, señala Stanchina (1975) en este mismo trabajo que "la autonomía es un experimento sobre cómo el aprendizaje puede liberarse de las ataduras de cualquier institución, y sobre cómo el individuo puede reclamar el control y la responsabilidad de su propia educación mientras investiga las oportunidades de aprender de una variedad de fuentes auténticas".

También Riley (1987) ve la autonomía como una capacidad, y más concretamente como "la capacidad de iniciar y dirigir con éxito el propio programa de aprendizaje". No obstante, la noción de 'responsabilidad' también está presente en sus afirmaciones, ya que considera al aprendiz el único responsable a la hora de tomar todas las decisiones relativas a la planificación y puesta en práctica de su programa de aprendizaje. Esta perspectiva tampoco difiere en gran medida de la ofrecida por Trim (1988: 3) en el prefacio a un documento publicado por el Consejo de Europa, según la cual lo que caracteriza a la autonomía es "la disposición y la capacidad de actuar de forma independiente como individuo social responsable, de hacerse cargo de las propias acciones y del propio aprendizaje al servicio de las propias necesidades".

Little (1990), por su parte, intenta clarificar el término desde la perspectiva de todo lo que la autonomía no es. No deberíamos dejar de señalar que este enfoque resulta bastante clarificador y puede ayudar en gran medida a desentrañar todos los malentendidos que han rodeado al término. Así, antes de llegar a su definición que ya anteriormente ofrecimos, Little dedica una sección titulada 'What autonomy is not' ('Lo que la autonomía no es') a enumerar todas aquellas falsas concepciones en torno al término, todo aquello que la autonomía no es. De este modo, para Little (1990: 7) los rasgos de lo que no es la autonomía podrían expresarse del modo siguiente:

- Autonomía no es sinónimo de 'auto-instrucción', es decir, de aprender sin un profesor, ya que la autonomía no es principalmente una cuestión de cómo se organiza el aprendizaje.

- En el contexto del aula la autonomía no requiere que el profesor decline toda la iniciativa y el control.

- La autonomía no es algo que los profesores hacen con sus alumnos, es decir, no se trata de una nueva metodología.

- La autonomía no es un comportamiento fácilmente describible ya que se puede manifestar de muchas formas.

- La autonomía no es un estado firme que alcanzan ciertos aprendices. Un aprendiz con un alto grado de autonomía en un área puede no ser autónomo en otra y, además, la permanencia de ese grado de autonomía no está garantizada.

En esta misma línea Waterhouse (1990) alude a diversos errores o malas interpretaciones de lo que implica la autonomía y que han tenido como resultado que el deseado 
objetivo de conseguir fomentar la autonomía en los aprendices, algo a lo que hoy en día pocos profesores se mostrarían contrarios, se tradujera en unos resultados reales harto decepcionantes. Algunos de estos errores a los que alude Waterhouse guardan un gran paralelismo con las afirmaciones de Little que acabamos de señalar acerca de lo que la autonomía no es, y se relacionan principalmente con el hecho de confundir la autonomía con un estado en el que los aprendices trabajan de forma aislada y en el que, por tanto, el papel del profesor se ve relegado a un segundo plano. De este modo, el profesor prestaría menos apoyo al aprendiz quizá por considerar que dicho apoyo supone una contradicción al creer que el aprendiz autónomo para poder ser considerado como tal debe ser 'abandonado a su suerte' a lo largo de su proceso de aprendizaje.

Existe, por tanto consenso entre los diferentes autores que han dedicado trabajos a analizar el fenómeno de la autonomía de aprendizaje. En primer lugar, dicho consenso se basa en el hecho de compartir la misma opinión acerca de las erróneas interpretaciones que se han hecho del término y que en muchas ocasiones han llevado a algunos a pensar que un aprendiz autónomo es aquél que trabaja solo, aquél que, siguiendo la metáfora de Holec (en Villanueva 1992: 37), es lanzado sin saber nadar a la parte profunda de la piscina. En segundo lugar, las diversas definiciones guardan como hemos visto una gran similitud entre sí, siendo quizá la ya mencionada de Holec (1981) la que mejor represente a todas además de ser la más utilizada en la literatura especializada.

Sin embargo, en los últimos años de la década de los noventa la perspectiva parece haber cambiado y se llegan a poner en entredicho los aspectos más importantes que de forma global parecían haberse convertido en indicadores de todo lo que la autonomía de aprendizaje lleva consigo: por una parte, lo que implica convertirse en un aprendiz autónomo y, por otra, la necesidad de contar con una definición clara y precisa del término autonomía.

Hasta ahora, de las definiciones de autonomía se desprendía como consecuencia lógica que un aprendiz autónomo era aquél capaz de hacerse cargo de su propio proceso de aprendizaje con todo lo que ello lleva implícito, siendo dicho aprendiz el único responsable de las decisiones tomadas en cuanto a objetivos, intereses, necesidades, material, ritmo y lugar de aprendizaje. Se aceptaba también que esta habilidad no es algo innato, sino algo para lo que hay que prepararse, perspectiva ésta que da como resultado el llamado learner training y que consiste en equipar al aprendiz con las destrezas necesarias para hacerse cargo de su propio proceso de aprendizaje, 'entrenamiento' éste que muchos autores consideran necesario para que un aprendiz consiga el estatus de autónomo (Dickinson 1988, 1992; Ellis y Sinclair 1989; Holec 1980; Little 1997; Villanueva 1994; Wenden 1991).

Sin embargo, cabría señalar que debido al cambio de perspectiva que, como acabamos de indicar, algunos autores adoptan en los últimos años, este entrenamiento o learner training es cuestionado por considerar que resulta contradictorio situar ambos términos ('autonomía de aprendizaje' y 'learner training') en un mismo contexto debido a las connotaciones de comportamiento automático que el último de ellos conlleva, así como por su influencia para que el aprendizaje autónomo quede reducido a una serie de destrezas (Esch 1997: 165). Tampoco Breen y Mann (1997: 134) consideran a la autonomía "un proceso que se puede aprender al igual que si se tratara de una serie de reglas y destrezas" 
y, como ellos mismos indican, difieren de Holec al proponer que la autonomía no es una aptitud o habilidad que tiene que ser aprendida, sino que ha de ser vista como "un modo de estar en el mundo; una posición desde la que comprometerse con el mundo" (p. 134).

La búsqueda de una definición homogénea del concepto de autonomía tan frecuente en muchos trabajos desde la década de los setenta (cf. Dickinson 1987; Holec 1981; Little 1990; Schwartz 1973; Trim 1988) tampoco se ha visto libre de críticas en algunos de los trabajos de estos últimos años, como es el caso de nuevo de Breen y Mann (1997), quienes se preguntan si realmente necesitamos tener una teoría unificada de la autonomía para que el concepto no se debilite. Para ellos es mucho más conveniente contar con diversas interpretaciones del término que lo enriquezcan. En sus palabras:

We suggest that the real issue is how different teachers are interpreting the concept in what they actually do in the classroom as an expression of their beliefs. We further suggest that a diversity of interpretations is actually preferable to some consensus definition, even if such were feasible. This diversity allows the innovation in action to reflect variability in cultural interpretations and classroom practices and, thereby, generates a multiplicity of alternative realizations (p. 149).

A la vista de estas posiciones tan encontradas, cabe preguntarse si realmente son excluyentes y si, por tanto, se ha de tomar partido por una u otra. Desde nuestro punto de vista, nos encontramos ante dos perspectivas entre las que se puede establecer un diálogo ya que el componente actitudinal tan presente en estas nuevas ideas no está ni mucho menos en contradicción con algo que, desde aquí seguimos defendiendo y que tiene que ver con el hecho de que saber aprender una lengua no es una competencia espontánea, sino que requiere una serie de destrezas que han de ser adquiridas mediante una formación en la autonomía.

\section{4. ¿POR QUÉ AUTONOMÍA?}

Como hemos podido apreciar en el apartado anterior, resulta complicado intentar buscar una definición consensuada del término autonomía, lo cual no debe desmotivar a todos aquellos que consideramos que la autonomía es una meta deseable por diversos motivos. Pero, ¿cuáles son esos motivos que justifican que el aprendiz sea quien debe asumir la responsabilidad en lo que concierne a su propio aprendizaje convirtiéndose, por tanto, en aprendiz autónomo capaz de aprender a aprender y, lo que es más importante, de aprender a pensar? De nuevo, la numerosa literatura sobre autonomía nos ofrece un amplio panorama al respecto, reflejando motivos más que suficientes para hacernos ver la importancia de que los aprendices se responsabilicen en mayor o menor grado de su propio proceso de aprendizaje. En dicha literatura los motivos encontrados son de variada naturaleza y diversos autores ofrecen diferentes clasificaciones de los mismos (Bosch, 1996; Dickinson, 1987; Little, 1990). Tomando a todos ellos como referencia podemos concluir que dichos motivos por los que la autonomía debe ser fomentada en los aprendices se pueden dividir en tres: i) motivos prácticos, ii) motivos psicológicos y iii) motivos educativos. 
Cuando hablamos de razones prácticas por las que la autonomía debe fomentarse hacemos referencia a aquellos motivos por los que una persona no lleva a cabo su aprendizaje en el contexto del aula. Henner-Stanchina y Riley (1978), hablando de aprendices adultos, reflejan dichos motivos, que incluyen la dificultad que pueden encontrar algunos de estos aprendices para asistir a las clases, bien sea por lejanía porque su vida familiar, social o profesional se lo impida o incluso por el hecho de que a algunos les desagrade el ambiente del aula (cf. Dickinson, 1987). Asimismo, es también frecuente encontrar a aprendices adultos cuyos objetivos de aprendizaje en la segunda lengua son muy concretos (cf. Dickinson, 1987) y a los que por tanto puede resultarles casi imposible encontrar una clase o un curso que de verdad les ayude a cumplir dichos objetivos. Por último, señalan Stanchina y Riley (1978), todos los aprendices tienen preferencia por unas técnicas y estrategias de aprendizaje concretas que el método aplicado por el profesor en el aula con toda probabilidad no les permitirá poner en práctica, lo cual seguramente hará que el curso pierda toda efectividad.

Blue (1981) va a cuestionar esa efectividad de los cursos tradicionales de idiomas y, aunque la situación a la que él hace referencia es una muy concreta (la de los postgraduados extranjeros de la universidad de Southampton), sin duda alguna puede hacerse extensiva a muchas otras. De hecho casi todas las razones que alega para afirmar la ineficacia de dichos cursos probablemente sonarán muy familiares a cualquier profesor de lenguas extranjeras. Así, como ejemplo, podemos señalar el gran número de alumnos por clase, la heterogeneidad de los grupos en cuanto a nivel de conocimientos, necesidades, ritmo de aprendizaje, sus diferentes consideraciones acerca de lo que lleva implícito aprender una lengua y, por supuesto, el factor inevitable de la creencia por parte de los aprendices de que el profesor es el único responsable de que el aprendizaje tenga lugar, rechazando así cualquier sugerencia que implique el más mínimo grado de responsabilidad por su parte.

Podemos, por tanto, resumir que aquellos que abogan por la idoneidad de un aprendizaje auto-dirigido lo hacen teniendo en cuenta, por un lado, factores personales (imposibilidad o dificultad por unos motivos concretos de acudir a clases regladas) y, por otro, factores que tienen que ver con aspectos que el aula lleva implícitos y que contribuyen a que sea muy difícil que un curso determinado se ajuste por completo a cada aprendiz.

Los motivos psicológicos por los que es positivo fomentar la autonomía en los aprendices tienen más que ver con la relación entre éstos y su proceso de aprendizaje. Aprender no es un proceso lineal ni acumulativo (Villanueva, 1992: 38), es decir, no es un proceso en el que los conocimientos, especialmente los lingüísticos, se van adquiriendo y acumulando uno tras otro sin que se establezca relación entre ellos y a su vez con los conocimientos ya existentes en cada uno de nosotros y que son los responsables de que cada uno asimile la información de una forma u otra.

En el marco del aula tradicional donde el aprendiz es dirigido en todo momento tanto por el profesor como por los materiales, sin la más mínima posibilidad de decidir qué es lo mejor para él en cuanto al programa, al ritmo de aprendizaje o al material a utilizar, se fomenta en gran medida en el aprendiz la idea de que no hay nada que él pueda aportar a su propio proceso de aprendizaje y que su único papel en el mismo es el de acumular aquellos conocimientos que el profesor y el programa le transmitan para más tarde en un 
examen demostrar que han sido adquiridos. Sin embargo, si uno de los principales objetivos de la autonomía es que la persona se convierta en individuo responsable en todas las facetas de la vida, ninguna institución estará capacitada para proporcionar todo el conocimiento necesario para ello, aunque sí podrá aportar la ayuda necesaria al aprendiz para que aprenda a pensar y a aprender y llegue a conocerse a sí mismo. De esa manera, conociéndose a sí mismo, llegará a ser consciente de que sus conocimientos previos (generales y particulares), así como su visión del mundo, son importantes y, como tal, los aplicará a cada nueva tarea de aprendizaje convirtiéndose así probablemente en un aprendiz más eficaz (Little, 1990: 8) que sabrá hacer de su aprendizaje el proceso de construcción que éste idealmente debe ser.

Por último, de la literatura podemos extraer una gran cantidad de motivos que podemos considerar educativos a favor de fomentar la autonomía de aprendizaje y que en su mayoría están relacionados con la diversidad en los aprendices. En los últimos años, y a partir del momento en que los procesos cognitivos del aprendiz pasaron a ser objeto de estudio, comienza a surgir una gran preocupación por conocer cómo determinadas características que diferencian a unas personas de otras pueden influir en su aprendizaje. Estas diferencias individuales entre los aprendices ${ }^{2}$ han sido objeto de numerosos trabajos (véase revisión en Brown, 1987; Ellis, 1994; Johnson, 2001; Skehan, 1989, 1991) al considerar los diferentes autores de forma prácticamente unánime que tales características individuales deben ser tenidas en cuenta cuando un aprendiz se enfrenta a la tarea de aprender una lengua.

De hecho, es bastante obvio para cualquier profesor de lenguas extranjeras que a todos sus alumnos no les gusta aprender de la misma forma (algunos prefieren explicaciones gramaticales mientras que otros sacan más partido de las clases más prácticas; los hay que prefieren ser corregidos a cada error que cometan cuando otros no desean ser corregidos en absoluto) y que a pesar de que el objetivo de cada lección sea puesto de manifiesto de forma clara por parte del profesor, el resultado en cuanto al aprendizaje por parte de los alumnos varía en gran medida y, para ello, basta, como indica Littlejohn (1985), con comprobar los diferentes resultados que en la evaluación final obtiene cada uno de ellos. Como bien señala Allwright (1988: 35) “Cada lección es una lección diferente para cada aprendiz".

Podríamos afirmar sin temor a equivocarnos que hoy en día ningún profesional del ámbito del aprendizaje de lenguas extranjeras ignora y/o resta importancia a la diversidad existente entre los aprendices. Indudablemente, el principal problema que se plantea, principalmente entre el profesorado, es la manera de afrontar dicha diversidad en las aulas $\mathrm{y}$, sin duda, un primer paso importante sería concienciar al aprendiz de la presencia de este factor diferenciador que más adelante le lleve a ser capaz de involucrarse y responsabilizarse en un aprendizaje que, sin duda, resultará mucho más eficaz.

\footnotetext{
2 Se han ofrecido muchas tipologías que recogen las diferencias individuales que pueden afectar al proceso de aprendizaje. Entre ellas, podemos destacar la de Altman (1980: 5-6) por ser una de las más completas, y que contempla las siguientes categorías principales de diferencias individuales entre los aprendices: i) edad, ii) sexo; iii) experiencia previa en el aprendizaje de una segunda lengua; iv) conocimiento de la lengua nativa; v) factores de personalidad; vi) aptitud para la lengua; vii) actitud y motivación; viii) ritmo de aprendizaje; ix) inteligencia; x) preferencia de modalidad sensorial (visual, táctil, etc.); xi) preferencia sociológica; xii) estilos cognitivos; xiii) estrategias de aprendizaje; xiv) errores.
} 


\section{CONCLUSIÓN}

No cabe duda de que la cuestión terminológica ha planteado y plantea dificultades en muchos ámbitos cuando no existe una definición clara y consensuada del concepto a utilizar.

En el caso que nos ocupa, hemos podido observar cómo el uso del término autonomía de aprendizaje no se ha visto exento de cierta polémica principalmente por tres motivos: en primer lugar por la existencia de diversos conceptos aparentemente destinados a la designación de un mismo fenómeno. En segundo lugar, debido a las erróneas interpretaciones que ha sufrido el término y, por último, a consecuencia del escepticismo de algunos autores que en los últimos años han llegado a cuestionar la necesidad de contar con una definición homogénea del concepto de autonomía.

No obstante y, a pesar de estas trabas, consideramos que a partir de todo lo expuesto se pueden extraer principalmente dos conclusiones básicas que nos ayuden a clarificar el tema tratado en este trabajo. Por una parte, la diversidad de términos utilizados en la literatura generalmente hacen referencia a dos ámbitos bien diferenciados como son la forma en que se organiza el aprendizaje y la competencia del aprendiz. Por otro lado, desde nuestro punto de vista, la importancia de lo que rodea a la autonomía no radica en la consecución de una definición homogénea y globalizadora del término, sino más bien en el entendimiento y puesta en práctica por parte del profesorado de todo lo que este término conlleva, de donde podemos destacar el cambio de papeles que se produce en las figuras del alumno y del profesor. En este sentido, el primero pasa a convertirse en principal protagonista de su proceso de aprendizaje involucrándose en la toma de decisiones de todos los aspectos que conforman dicho proceso y, el segundo, es decir el profesor, a convertirse en un facilitador de este proceso dejando a un lado su papel de figura 'autoritaria' que durante tanto tiempo ha ejercido en el aula.

\section{REFERENCIAS BIBLIOGRÁFICAS}

Allwright, R. 1988. "Autonomy and individualization in whole-class instruction", Individualization and autonomy in language learning. Eds. A. BRoOKes \& P. GRUNDY ELT Documents, 131. London: Modern English Publications/The British Council, $35-44$.

BLUE, G.M. 1981. "Self-directed learning systems and the role of the ESP teacher. The ESP teacher: Role, development and prospects". ELT Documents 112, 58-64.

Bosch, M. 1996. Autonomia i aprenentatge de llengües. Barcelona: Graó.

Breen, M. \& MANn, S. 1997. "Shooting arrows at the sun: Perspectives on a pedagogy for autonomy". Autonomy and independence in language learning. P. BENSON \& P. VoLLER, eds. London: Longman, 132-149.

Brown, H.D. 1987. Principles of language learning and teaching. Englewood Cliffs: Prentice Hall. 
CARver, D.J. \& Dickinson, L. 1982. "Learning to be self-directed". Individualisation. Eds. M. Geddes \& G. Sturtridge. Londres: Modern English Publications, 15-21.

DAM, L. 1990. "Learner autonomy in practice". Autonomy in language learning. Ed. I. Gathercole. London: CILT, 16-37.

Dickinson, L. 1976. Self-directed learning and autonomy. Cambridge: Cambridge University Press.

186.

. 1979. "Self-instruction in commonly taught languages". System 7, 181University Press.

. 1988. "Learner training". Individualization and autonomy in language learning. Eds. A. Brookes \& P. Grundy. ELT Documents 131. London: Modern English Publications / The British Council, 45-53.

ElLIS, R. 1994. The Study of Second Language Acquisition. Oxford: Oxford University Press.

Esch, E. 1997. "Learner training for autonomous language learning”. Autonomy and independence in language learning. Eds. P. BENSON \& P. Voller. London: Longman, 164-176.

Henner-Stanchina, C. y Riley, P. 1978. “Autonomous learning”. Individualisation in language learning. ETIC Document, 103. London: The British Council.

Holec, H. 1980. "Learner training: Meeting needs in self-directed learning”. Foreign language learning: Meeting individual needs. H.B. Altman y C. Vaughan James, eds. Oxford: Pergamon, 30-45.

.1981. Autonomy in foreign language learning. Oxford: Pergamon.

1985. “On autonomy: Some elementary concepts". Discourse and learning. Ed. P. RILEY. London: Longman, 173-190.

ILLICH, I. 1971. Deschooling society. New York: Harper y Row.

JoHnson, K. 2001. An introduction to foreign language learning and teaching. Harlow: Longman.

Knowles, M. 1975. Self-directed learning: A guide for learners and teachers. New York: The Adult Education Company.

Little D. 1990. “Autonomy in language learning”. Autonomy in language learning. Ed. I. Gathercole. London: CILT, 7-15.

. 1997. "Language awareness and the autonomous language learner". Language Awareness 6: 93-104.

LitTLEJohn, A. 1985. 'Learner choice in language study'. ELT Journal, 39, 253-261.

Logan, G.E. 1973. Individualised foreign language learning: An organic process. Rowley Mass: Newbury House. 
RILEY, P. 1987. "From self-access to self-direction”. The Advanced Language Learner. Eds. J.A. Coleman y R. Towell. London: CILT, 75-88

Schwartz, B. 1973. L'Education Demain. Une Étude de la fondation Européenne de la Culture. París: Aubier Montaigne.

Stanchina, C. 1975. "The logic of autonomy as a strategy for adult learners". University of Nancy, CRAPEL: Mimeo.

1976. "Autonomy at work with adults. Self-Directed Learning and Autonomy. Ed. E. Esch. Cambridge, Departamento de Lingüística: Universidad de Cambridge, 35-65.

Sturtridge, G. 1982. "Individualised Learning: What are the Options for the Classroom Teacher?". Individualisation. Eds. M. Geddes y G. Sturtridge. London: Modern English Publications, 8-14.

TRIM, J.L.M. 1988. "Preface". Autonomy and self-directed learning: Present fields of application. Ed. H. Holec. Estrasburgo: Consejo de Europa.

Tumposky, N. 1982. "The learner on his own”. Individualisation. M. Geddes y G. Sturtridge, eds. London: Modern English Publications, 4-7.

Villanueva, M.L. 1992. "El aprendizaje de la autonomía: Desarrollo de la conciencia lingüística”. Ici \& Lá 22: 37-41. . 1994. "Un proyecto de diálogo entre la enseñanza-aprendizaje en situación de clase y el aprendizaje en los C.A.L.". Nuevos horizontes de la lingüística aplicada. Eds. J. Llisterri y D. Posch. Actas del XII Congreso Nacional de AESLA. Universidad Autónoma de Barcelona, 409-416.

Waterhouse, P. 1990. “The curriculum”. Autonomy in Language Learning. Ed. I. GATHERCOLE. London: CILT, 4-6.

WENDEN, A. 1991. Learner strategies for learner autonomy. London: Prentice Hall. 